\title{
Web 2.0 and Participatory Governance
}

\author{
Peter Towbin \\ Economics Department, University of California, Santa Cruz, Ca. USA. ptowbin@gmail.com \\ Yongjun Zhang \\ Geography and Resource Management, The Chinese University of Hong Kong. yjzhang@cuhk.edu.hk
}

\begin{abstract}
By integrating a Geographic Information System (GIS) into a web portal, we allow a multi-way dialog between Hong Kong's citizens and planning officials. Alternative development plans for Lantau (Hong Kong's largest island) can be analyzed through interactive maps, which allow citizens to compare and comment on specific geo-referenced features. Lantau Island's extensive nature reserves, which offer protected nesting grounds for numerous bird species and other ecological and recreational services, are being weighed against extensive economic development. This experiment in open governance within China will also serve as a laboratory to study qualitative differences in citizen learning, between online dialog and face-to-face group deliberation. Our experiments will explore resolutions to a classical economic paradox from social choice theory, and point to potential improvements in contemporary efforts to bring open and responsive government through information technology.
\end{abstract}

Keywords: Lantau Island, Hong Kong, geographic information system, GIS, deliberation, social choice theory.

Acknowledgement: We would like to acknowledge the University of California's Institute on Global Conflict and Cooperation and Pacific Rim Research group for their generous support that has made this collaboration possible.

r

he quest for opportunity through economic growth, versus the preservation of our ecological heritage and natural beauty, form the centerpiece of many contemporary public debates. Innovations in web technology provide unprecedented opportunity for citizen engagement in forming public policies, which must make tradeoffs between these oft competing goals. Hong Kong, which is itself uniquely positioned in the history of local versus central control, is the setting for deployment of our participatory geographic information system (PGIS): The Lantau Public Consultation web site.

Lantau is the largest island of the Hong Kong Special Administrative Region of China. The government of Hong Kong, which has a high degree of autonomy from the mainland (at least until 2047), is in the process of revising its long range plan for Lantau Island's development, and they have undertaken a number of efforts to solicit public opinion concerning development options, such as opinion surveys and public discussion forums. The Public Consultation web site incorporates state-of-the-art GIS tools to enable citizens to explore, analyze, and comment on government proposals through a map-based interface, with user-defined geographic references. But they also engage fundamental theoretical controversies in collective decision making and the role of government.

Through this collaboration between the Chinese University of Hong Kong (CUHK) and the University of California, Santa Cruz, we will gain insight into both contemporary and classical questions. Yongjun Zhang is principally responsible for the web PGIS system. Peter Towbin designed a series of experiments to understand the potential roles for Web 2.0 in participatory governance and examines the connection to one of the continuing debates of modern economics, embodied in Kenneth Arrow's impossibility results for social choice theory (Arrow, 1951). 


\section{Lantau PGIS}

The Lantau Public Consultation web site synthesizes a number of ideas into one participatory geographic information system. Its back-end is built from ESRI's arcGIS server, which is the industry standard tool for government planning agencies around the world. The server stores detailed topographic information about Lantau Island, as well as representations of alternative government proposals and user defined features and comments. The web interface integrates Google Maps for geographic browsing and search, citizen dialog through geo-referenced discussion threads, and topographic analysis of how the various plans impact specific land-use regions.

A great deal of work has gone into codifying regions of Lantau (in the GIS database) for their environmental and historic value, as well as their business potential. There will be four or five alternative plans presented, which highlight different preference attitudes. Citizens will be encouraged to enter a preference profile, which gives a generic measure of how they value different land use priorities. They will also be able to rate the specific plans. Methodologies for rating environmental valuation are quite controversial, since they involve judgment of hypothetical situations (List and Gallet, 2001). One of our contributions to the valuation literature will be to use this data to calibrate various scoring metrics for placing the different plans in a multi-dimensional preference landscape.

The Chinese University of Hong Kong's motto is "To Combine Tradition with Modernity, To Bring Together China and the West". By combining home-grown innovation with collaboration from the University of California, and giving voice to citizens' own priorities for preserving tradition versus modernization, the Lantau PGIS does exactly this. And it comes at a timely moment. The ongoing transformation and opening up of Chinese society has advanced in fits and starts, driven by smallscale experimentation that spreads by demonstrating its value. At this moment, China's central government is concerned with the spread of interactive mapping technology and has passed legislation to strengthen central oversight (Xinhua News Agency, 2007). We believe that drawing attention to constructive applications of citizen mapping, as in the Lantau PGIS, is the best way to support the long-term evolution of policy.

\section{Participatory Policy meets Decision Theory}

Decision theory tends to take a rather abstract view of problem solving. It can indicate the optimal solution, but only when the problem can be formulated with explicit mathematical functions. The standard approach models individuals in the tradition of economic theory, as "rational agents". Agents are represented through their "utility function", which maps possible outcomes onto a linear preference relation. This is the "consistency requirement" of subjective expected utility (SEU) theory: if you prefer A over B, and B over C, then you are required to prefer A over C.

Unfortunately, real humans violate this and other postulates of SEU much more often than theorists had anticipated (Camerer, 2003). But, in designing a tool to support decision making, one needs some criterion for selecting one option over another, and we are led inexorably back to the idea of maximizing some sort of expected utility ${ }^{1}$.

But, even if we treat individuals as optimal rational agents, we hit another fundamental problem when we try to scale up our utility function from the individual to the group. Suppose we have perfect knowledge of each individual's preferences, in comparing all options: Person 1 prefers option $\mathrm{Z}$ over $\mathrm{B}$, has equal preference for $\mathrm{B}$ and $\mathrm{C}$, prefers $\mathrm{C}$ over $\mathrm{Y}$, and so on, for each person.

\footnotetext{
${ }^{1}$ We can only maximize "expected'" utility, since their are facts we don't know with certainty, such as whether it will rain this afternoon. So, even though we would much rather have an umbrella when it is raining, we would leave it at home if we expect it will not rain -- judged by our subjective evaluation of the probability of rain.
} 
But, when we apply some algorithm to merge these preferences into a "group preference" statement, the merged set may no longer satisfy the SEU consistency requirement mentioned above. Kenneth Arrow's "Impossibility Theorem" (Arrow, 1963) proves that, no matter what algorithm is used, there will be some input such that the group utility has preference cycles, such as: the group prefers $\mathrm{A}$ over $\mathrm{B}, \mathrm{B}$ over $\mathrm{C}$, but then prefers $\mathrm{C}$ over $\mathrm{A}$ !

"Social choice theory" refers to the very extensive literature that has grown around this issue, and its significance goes far beyond the algorithmic realm. The same theoretical assumptions can derive a theory that market mechanisms do produce optimal outcomes purely through the aggregate effects of (SEU rational) individual decisions. The combination of these two results forms the foundation of a prominent libertarian philosophy that argues against the legitimacy of almost any form of government planning efforts (Riker, 1982). The argument is that a group decision-making body such as the Congress is unable to guarantee a linear preference ordering, and thus, is not rational. And it is better to delegate to an optimal market than an irrational government.

However, recent work has given some empirical results indicating that the problem uncovered in Arrow's theorem can be mitigated: through a structured, participatory process, involving face-toface dialog. Our experiment will explore the basis of this phenomena, to better understand its significance, and to see what lessons can be transferred to systems that could accommodate large-scale participation -- such as the Lantau PGIS.

\section{Bayesian Modeling and Collective Decision}

Traditional statistical methods are concerned with evaluating hypotheses with respect to a body of data. In contrast, Bayesian statistics considers an individual who may already have a sense for the probability that a hypothesis is true, garnered from past experiences. This is called the "prior" probability, and it is a subjective assessment by that individual. Bayesian theory describes the optimal way to revise one's prior belief, given a new body of data. In fact, the Bayesian approach has been adopted as the normative strategy for belief revision in the rational agent paradigm.

In order to put decision theory to work on real world problems, prior probabilities play a key role in bridging the gap between the idealized rational agent and ordinary human agents. We can ignore whatever tortuous path led the user to his/her current beliefs, and focus on helping them incorporate new information appropriately. In the case of natural resource management, sometimes we have the more modest goal of simply trying to build a reasonable model of the human component of the system dynamics. However, successful management often requires that local actors also gain a deeper understanding of the workings of the system, and their own impact upon it. Bayesian networks comprise one modeling approach that has proven quite useful in both cases, and there is a rapidly growing literature on their application in participatory resource management scenarios (Cain et al, 1999; Cain et al, 2001; McCann et al, 2006).

Bayesian networks have two properties that make them particularly useful for representing human cognitive models. Statistical methods are often restricted to looking at correlations between events, without commenting on what might cause the correlations. Bayesian networks are designed explicitly to represent causal relations, just as the mental models that humans construct internally are more often about causality than about mere correlation. The second factor is that these networks can be depicted very intuitively as a directed graph. Imagine two variables of interest, for example, $\mathrm{S}=$ the size of a new housing development, and $\mathrm{B}=$ the number of surviving hatchlings in a neighboring bird nesting ground. Then, $S$ and $B$ would be represented as nodes in the network, and you would draw an arrow from $S$ to $B$-- if you believe the housing project will have a causal impact on the number of surviving birds.

Accurate and objective modeling of environmental dynamics is hard. When humans are an intimate part of those dynamics, it is impossible: human behavior is guided by inherently subjective beliefs, and those are generally conditioned on beliefs about the future actions of other humans -beliefs about beliefs. Participatory modeling and policy development is driven by the need to bring 
the humans into the equations, to get as accurate an account of how they would respond to alternative scenarios as possible. In the literature we have surveyed, stakeholder engagement and belief elicitation play a key role in building a Bayesian network model of the environmental system. In contrast, we are not looking for the single best statistical model of system behavior. We want a representation of each participant's individual beliefs, however divergent they may be from reality or each other. Communities contain a diverse population of individuals, with different roles, aspirations, and histories. Our goal is to accurately represent this diverse community of beliefs.

\section{An Experiment in Deliberative Polling}

Crafting productive civic engagement does not have to be easy to be possible. If a feckless drift toward polarization of group opinion was made inevitable by the process of discussion, much of the current debate would have to be rethought. But, studies indicate that this danger can be mitigated through "deliberative" group process: a carefully facilitated group dialog, which focuses on balanced presentation of positions, and the task of understanding viewpoints rather than choosing from them (Gastil, 2004; Hamlett, 2006). But the time intensive nature of participatory methods remains an issue. As the argument from democratic theorists for an increased level of civic engagement and political participation by average citizens becomes more insistent (Barber, 1996), so do the distractions of our networked world. Fortunately for the many issues that merit careful public consultation, but cannot justify large-scale public involvement, there is the ingenious method of "deliberative polling", developed by Jim Fishkin of the Stanford Center for Deliberative Democracy.

Early reforms to promote openness in government and civic engagement led to town-hall style meetings, and opportunities for citizens to address city councils and other government bodies. This has increased the exposure of public officials to the opinions of citizens. But the citizens who most often participate are representatives of competing interest groups. The opinions sampled are highly informed but also highly unrepresentative of the general public. On the other hand, public opinion polls offer a randomized sampling of the population. But for complex, interwoven problems that require thoughtful consideration, an unbiased sampling of off-the-cuff opinions from a telephone survey is a serious mismatch. In a deliberative poll, a random sampling is done to find a (smaller) group of citizens who are willing to participate (generally, with monetary compensation) in a group deliberative dialog event. Ample evidence shows that deliberation makes a difference -opinions on substantive issues sometimes shift significantly during the course of deliberation (Fishkin, 2008).

Another more tentative result is quite intriguing: one study found that the process of deliberation re-aligns individual preference profiles in such a way that Arrow's impossibility result is less likely to hold (Farrar et al, 2003). But, so far, these studies have only looked at changes to preference profiles. Our experiment will compare two groups of citizens using the Lantau PGIS. The first group will learn individually from the Lantau PGIS web site. The second group will initially have the same individual learning time with the Lantau PGIS, but will subsequently meet for a face-to-face deliberative dialog.

By comparing changes in preference structures, we will provide more evidence as to the strength of the preference alignment phenomena. And by eliciting Bayesian network representations from the participants, we will see if the change derives from substantive learning about the causal structure of the problem or something more akin to "emotional contagion" (Barsade, 2002). Although alignment through substantive learning has a more noble ring to it, a strong effect for either result could have significant implications for the evolution of web based public consultation and governance systems.

\section{References}


Arrow, K. (1951). Social Choice and Individual Values. New York, NY: Wiley.

Barsade, S. (2002). “The Ripple Effect: Emotional Contagion In Groups" Administrative Science Quarterly, Vol. 47.

Camerer, C., (2003) Behavioral Game Theory. Princeton University Press, Princeton, New Jersey.

Cain, J., \& Batchelor, C., \& Waughray, D. (1999). Belief Networks: A framework for the participatory development of natural resource management strategies. Environment, Development and Sustainability, 1, pp. 123-133.

Cain, J. (2001) "Planning improvements in natural resources management: Guidelines for using Bayesian networks to support the planning and management of development programs in the water sector and beyond" Wallingford, UK: Centre for Ecology and Hydrology.

Farrar, C., \& Fishkin, J. S., \& Green, D. P., \& List, C., \& Luskin, R. C., \& Paluck, E. L. (2003). Experimenting with Deliberative Democracy: Effects on Policy Preferences and Social Choice, Paper presented at the ECPR conference in Marburg, Germany. Retrieved August 2008, from Ilcdd.stanford.edu/research/papers/2003/experimenting.pdf.

Fishkin, James S. "Deliberative Polling: Toward a Better-Informed Democracy" Center for Deliberative Democracy. Stanford University. Retrieved August 2008, from Ilcdd.stanford.edu/polls/docs/summary.

Gastil, J. (2004). “'Adult civic education through the National Issues Forums: A study of how adults develop civic skills and dispositions through public deliberation" Adult Education Quarterly, 54, pp308-328.

Hamlett, P. W., \& Cobb, M. D. (2006). " Potential Solutions to Public Deliberation Problems: Structured Deliberations and Polarization Cascades" Policy Studies Journal 34 (4), 629-648.

List, J., \& Gallet, C. (2001). "What Experimental Protocol Influence Disparities Between Actual and Hypothetical Stated Values?" Environmental and Resource Economics. vol. 20, No. 3 pp. 241-254, 2001.

McCann, R. K., \& Marcot, B. G., \& Ellis, R. (2006). “Bayesian belief networks: applications in ecology and natural resource management" Canadian Journal of Forest Research, 36(12), pp3053-3062.

Riker, W. (1982). Liberalism against populism: A confrontation between the theory of democracy and the theory of social choice. Prospect Heights, IL: Waveland Press.

Sunstein, C. R. (1999). "The Law of Group Polarization". University of Chicago Law School, John M. Olin Law \& Economics Working Paper No. 91.

Xinhua News Agency. (2008) "China cracks down on illegal online map services to protect state security" Retrieved March 3, 2008, from verb|news.xinhuanet.com/english/2008-03/25/content_7858467.htm|

\section{About the Authors}

Peter Towbin

Peter Towbin is a Ph.D. student at the department of Economics, University of California, Santa Cruz. He received a Masters degree in Computer Science from the University of California, Irvine, and a Bachelors degree in Mathematics from the University of California, Santa Cruz.

\section{Yongjun Zhang}

Yongjun Zhang is a Ph.D. student at the department of geography and resource management, the Chinese University of Hong Kong. His research interests include Public Participation GIS, Spatial Decision Support System, and Environmental Management. He got his bachelor degree in Environmental Engineering from the University of Science and Technology, Beijing. And then he obtained his Masters degree in Environmental Science from Beijing Normal University. 